\title{
PET/MR attenuation correction in brain imaging using a continuous bone signal derived from UTE
}

\author{
Claes Ladefoged", Didier Benoit, lan Law, Soren Holm, Liselotte Hojgaard, Adam Espe Hansen, \\ Flemming Littrup Andersen
}

From PSMR 2015: 4th Conference on PET/MR and SPECT/MR

La Biodola, Isola d'Elba, Italy. 17-21 May 2015

Department of Clinical Physiology, Nuclear Medicine and PET, Rigshospitalet Copenhagen, Copenhagen, Denmark
In the absence of transmission sources in combined clinical PET/MR systems, MR images are used for MR-based attenuation correction (MRAC). The main challenge in MR-AC is to separate the bone and air, as neither have a signal in the MR images. In the attenuation maps supplied by the vendor, a single value is assigned to bone using an ultra-short echo time (UTE) MR sequence. The purpose of this study was to develop a new multi-class segmentation-based MR-AC method, employing Continuous-Bone-using-R2* (MRAC_CBuR2*), and evaluate it on a large patient cohort. 53 [18F]-FDG PET/MR brain patients were included in this study. MRAC was based on an aligned CT (MRAC_CT, used as reference), standard MRAC_UTE and MRAC_CBuR2*. Our method segments the air, brain, CSF and soft tissue voxels on the UTE images, and uses a mapping of $\mathrm{R}^{*}$ values to HU to measure the density in bone voxels. Aligned anatomical masks are used to improve accuracy in noisy regions. Region-based analysis was performed using ICBM 2009a brain atlas with anatomical labels pre-defined. Using CBuR2 $*, 82 \%$ of the voxels in the brain are within $\pm 5 \%$ of PET_CT, compared to $27 \%$ when using UTE. Using our method, there are clear improvements over UTE. The average error over the full brain is $0.8 \%( \pm 1.7 \%)$, compared to $-7.1 \%( \pm 2.4 \%)$ in UTE. Of note, the maximum error in the cerebellum is $-15 \%$ and $7 \%$ in UTE and CBuR2*, respectively. The proposed method uses the available UTE images to segment tissue classes, and uses the R2* map to measure a continuous bone signal. The improvement over the vendor provided UTE reduces both the global and local error on the reconstructed PET images.

Published: 18 May 2015

doi:10.1186/2197-7364-2-S1-A39

Cite this article as: Ladefoged et al:: PET/MR attenuation correction in brain imaging using a continuous bone signal derived from UTE. EJNMMI Physics 2015 2(Suppl 1):A39.

\section{SpringerOpen $^{\odot}$}

(c) 2015 Ladefoged et al; licensee Springer. This is an Open Access article distributed under the terms of the Creative Commons Attribution License (http://creativecommons.org/licenses/by/4.0), which permits unrestricted use, distribution, and reproduction in any medium, provided the original work is properly cited. 